¿Pero qué es el "lenguaje integrado?

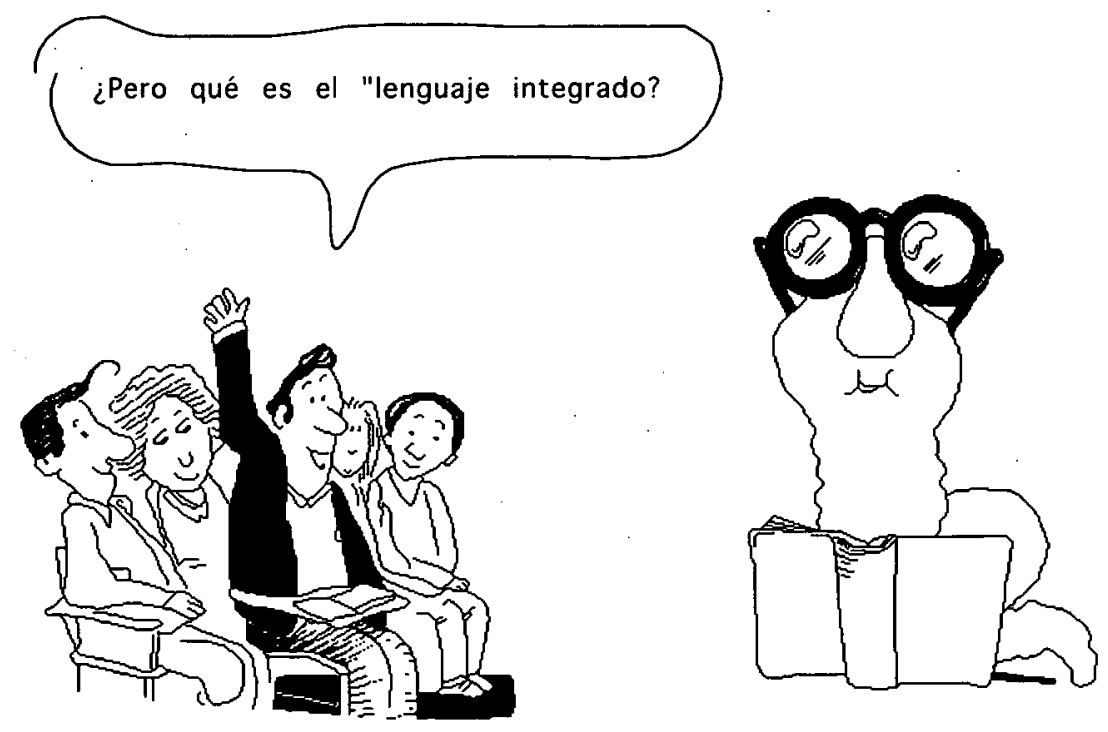




\section{Lenguaje integrado: ¿simple práctica, un método o una filosofía?}

\section{Pilar Lacasa, José Jerónimo Anula y Beatriz Martín}

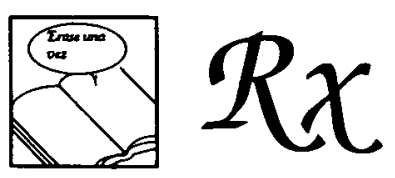

¿Qué es realmente el movimiento conocido como "lenguaje integrado"?, ¿ se trata sólamente de un conjunto de prácticas que ban adoptado en el aula un número cada vez más numeroso de enseñantes?, ¿cuáles son los principios en los que se apoya?, ¿qué relación existe entre este movimiento y otras aproximaciones innovadoras en educación? Este articulo es un intento de responder estas y otras cuestiones.

Una de las tareas más importantes de la escuela es enseñar a los niños, desde muy pequeños y casi desde el primer día en que se incorporan a ella, a leer y escribir. Descifrar y producir los códigos del lenguaje escrito, saber expresarse en ese medio, servirse de él cuando es necesario, se han convertido en una habilidad imprescindible en las sociedades occidentales. Quizás desde esta idea se comprenda mejor qué es lo que en el mundo anglosajón se conoce como «whole language» y nosotros hemos traducido como lenguaje integrado. No cabe duda de que el término y, por qué no, su traducción al castellano son atractivos.

\section{¿QUÉ ES EL MOVIMIENTO CONOCIDO COMO LENGUAJE INTEGRADO?}

Pero ¿qué es realmente este movimiento? Es difícil encontrar una definición en la que educadores, investigadores o incluso quienes se ven obligados a planificar el sistema educativo se pongan de acuerdo. Podemos comenzar con la siguiente:

«Lenguaje integrado es un concepto que incluye tanto una filosofía del lenguaje como como una aproximación instruccional enmarcada y apoyada por esa filosofía. Ese concepto incluye el uso de la la literatura y la escritura real en el contexto de experiencias significativas, funcionales y de cooperación, con el fin de desarrollar en los estudiantes la motivación y el interés durante el proceso de aprendizaje.» (Bergeron, 1990, p. 319)

Tres aspectos cabe destacar de esta definición. En primer lugar, estamos ante una filosofía, un conjunto de presupuestos desde los que nos acercamos a las situaciones educativas y que se refieren, por una parte, a la naturaleza del lenguaje y a su 
papel en el currículum y, por otra, a las características que definen las actividades de quienes enseñan y aprenden en la escuela (Goodman, 1992/Noviembre). En este contexto este movimiento valora el aula como una comunidad democrática de aprendizaje donde los maestros y los alumnos aprenden juntos.

En segundo lugar, el lenguaje integrado nos acerca a una determinada práctica educativa. De acuerdo con Goodman y Goodmann (1990) es «un movimiento, holista, dinámico y popular entre los maestros» que se ha extendido, sobre todo, en los paises anglosajones. En Inglaterra, por ejemplo, aunque el término lenguaje integrado no es realmente tan popular, los principios en que se apoya son similares a los que se defienden en movimientos como "el lenguaje a través del currículum» u otras prácticas de carácter holista. Es bien conocido también en Australia, en Estados Unidos y en Canada.

Por último, en tercer lugar, sus fines se relacionan directamente con la necesidad de crear en el que aprende una actitud positiva, un mayor interés hacia el aprendizaje. de integrar

En la figura 1 incluimos un conjunto de conceptos que este movimiento trata

FIGURA 1

Algunos conceptos educativos presente en la filosofía del Lenguaje integrado

(a partir de Goodman, 1992)

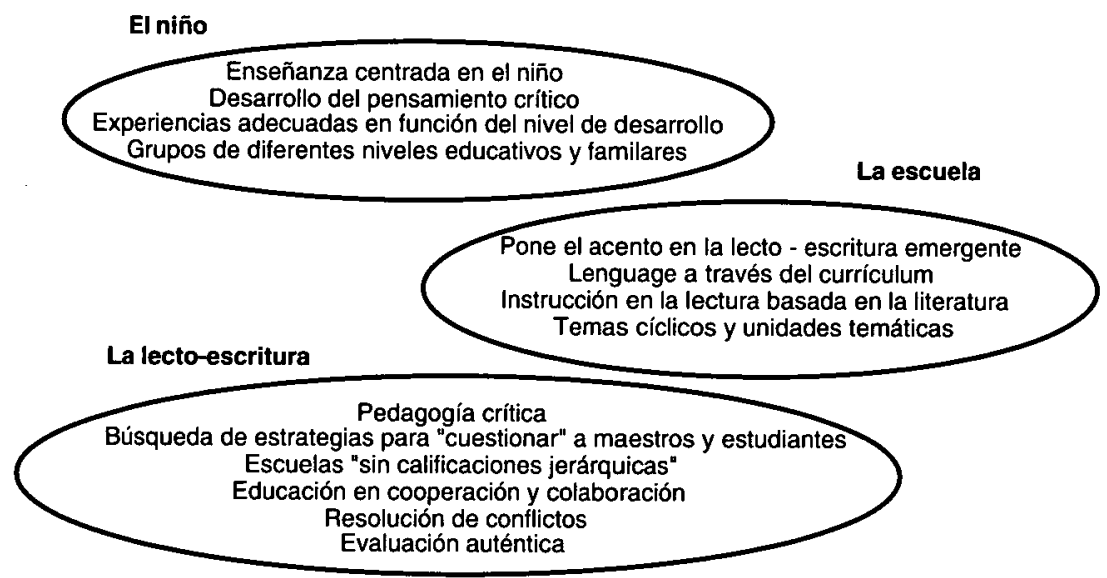

\section{¿CUÁLES SON SUS PRESUPUESTOS?}

Pero, ¿cuáles son las bases de esa filosofía?, ¿por qué lenguaje integrado? Construir el significado y acercarse al lenguaje desde un enfoque funcional puede considerarse su punto de partida. Nos referiremos ahora a los principios que se aceptan como básicos, los organizaremos en torno a la naturaleza del lenguaje y a lo principios que rigen su desarrollo y aprendizaje (Goodman \& Goodmann, 1990). Veremos, además, en relación con cada uno de ellos, sus aplicaciones más inmediatas en el aula.

1) El lenguaje es una actividad centrada en el significado (Long, Wolfson, Manning \& Manning, 1987). Para quienes se sitúan en esta perspectiva leer y escribir significa comprender. Las auténticas experiencias relacionadas con la lecto - escritura son acontecimientos que tienen un significado personal para quien utiliza el lenguaje. 
En este sentido, existen intercambios entre el lector y el texto, en los que el lector está continuamente resolviendo nuevos problemas y construyendo y ampliando estrategias psico - lingüísticas. En esos intercambios el texto sirve de mediador en el desarrollo de la lectura y la escritura. La definición de texto que se acepta es la que proponen Halliday y Hasan (1976), de acuerdo con la cual el texto es la unidad semántica básica de interacción lingüística. Si nos fijamos en las consecuencias que de ello se derivan para la práctica educativa, veremos que las clases se estructuran en torno a actividades significativas, por ejemplo diarios, discusiones de grupo, etc. cuyo contenido temático es elegido por los alumnos. Estas actividades sustituyen a otras que tienen lugar en clases segmentadas entre dictados, lectura, ejercicios, etc. y que se destinan exclusivamente a la adquisición de habilidades donde el significado natural del lenguaje se desvirtúa.

2) El lenguaje, incluido el lenguaje escrito, se aprenden más fácilmente en el contexto de su uso y en relación con las metas que se generan en esos contextos. "Cuando el lenguaje es un todo relevante y funcional, el que aprende tiene metas reales para usarlo (Goodman y Goodmann, 1990, p. 225). La lectura y la escritura tienen por ello un objetivo comunicativo que se enfatiza en las actividades o episodios educativos (Brazee y Kristo, 1989). De ahí que en las clases planificadas desde la filosofía de lenguaje integrado se generen situaciones en las que los alumnos están dirigidos hacia metas significativas relacionadas con el uso del lenguaje (Altwerger, Edelsky, \& Flores, 1987/Noviembre), por ejemplo, leer un periódico, escribir las propias opiniones para elaborar un artículo, inventar una novela, etc.

3) Leer, escribir, bablar y escuchar son diferentes aspectos de lenguaje que ban de considerarse de forma integrada (Manning y Manning, 1989). Así, dimensiones como la fonología en el lenguaje oral, la ortografía en el escrito, la morfología, la sintaxis, la semántica y la pragmática están siempre simultáneamente presentes e interactuan en cualquier aspecto del lenguaje en uso. Por ello; para que el proceso de aprendizaje no interrumpa esta interdependencia, las clases se estructuran en torno a un contenido significativo para los alumnos y éste es abordado desde la lectura, la escritura y el habla (Butler, 1991). Los diferentes aspectos del lenguaje quedan conectados por los mismos significados ya que se habla sobre lo que se lee y sobre lo que se escribe y se escribe sobre lo que se lee o sobre lo que se habla.

4) El lenguaje se aprende primero de forma integrada para irse diferenciando y refinando posteriormente. Este supuesto se traduce en una opinión en contra de la enseñanza del lenguaje de forma fragmentada. Para muchos autores (por ejemplo, Adams, 1990; Chall y Curtis, 1992; Walberg, 1992) un aspecto realmente importante del lenguaje integrado es el rechazo a la instrucción fónica, una práctica de acuerdo con la cual el lenguaje se segmenta fonéticamente. Se apoyan en la idea de que los bebes aprenden a hablar utilizando la lengua en situaciones concretas y dentro de una comunidad que le da sentido (Linfords, 1987; Goodman, 1989; Weaver, 1990; Allen y col. 1991). Desde esta perspectiva, la lectura y la escritura son vistas como procesos y no como una acumulación de pequeñas destrezas. Por ello, en las clases los niños son aproximados de forma integrado a amplias unidades de significado (Harp, 1988) para que, a partir de la práctica, el aprendizaje sea posteriormente refinado respondiendo a unidades de significado menores (Froese, 1991) como frases, palabras y finalmente deletreos y correspondencias fonema-grafema.

5) El lenguaje se aprende a través de la práctica. En este contexto se asume que las destrezas lingüísticas como mejor se aprenden es a través de su uso y no mediante una enseñanza descontextualizadora que se considera artificial. Esa práctica no es ajena al entorno social en que se produce. Los que aprenden aportan conocimiento al proceso de construcción del significado, ese conocimiento y las relaciones entre la personas, así como el entorno mismo, influyen en la facilidad con que se desarrollan 
los procesos lingüísticos. El lenguaje y el pensamiento del niño tienen sus raíces en el hogar y la comunidad, de ahí la necesidad de que los padres formen parte de la comunidad educativa.

6) Hay que reconocer también el importante papel que desempeña el lenguaje en el aprendizaje humano y este principio puede condensarse en la idea de Goodman y Goodmann (1990) «aprender el lenguaje y aprender a través del lenguaje». El lenguaje hace posible compartir la experiencia, unir nuestras mentes y producir una inteligencia social que es superior a una individual. Podemos aprender a través de experiencias vicarias compartidas a través del lenguaje. El lenguaje, oral o escrito, no puede ser sólo un tema escolar. Además, cuando la lengua se utiliza en las escuelas, los estudiantes oyen, leen, hablan y escriben acerca de los contenidos de diversas áreas del currículum y, por otra parte, los maestros comprenden que la interpretación de los conceptos básicos del contenido de esas áreas precede, o es paralelo, a la interpretación de la palabras que son peculiares de dominios específicos de conocimiento. Por todo ello los maestros valoran, respetan y hacen uso del contenido de las áreas del currrículum para promover el lenguaje oral y escrito (Watson, 1989/Noviembre).

7) El aprendizaje del lenguaje supone la interacción y contraste de dos fuerzas, centrípetas y centrifugas que conforman el desarrollo lingüístico en los individuos y la comunidad (Goodman y Goodmann, 1990). Esas fuerzas, aunque se oponen, operan de forma conjunta y coordinada. Por fuerza centrifuga se entiende la habilidad individual y colectiva de las personas para crear sistemas semióticos, inventar un nuevo lenguaje, hacer frente a nuevas experiencias sentimientos e ideas. Esa fuerza hace posible que el lenguaje se desarrolle y cambie a través de sus usuarios. La fuerza centrípeta aporta un contrabalanceo a esas fuerzas e introduce una relativa estabilidad en la naturaleza social del lenguaje. Se trata, por tanto, de establecer un equilibrio entre invención y convención. Ambos, la invención, entendida como la creación personal del lenguaje, y la convención, definida como sistema establecido y conjunto de normas, son necesarias en el aprendizaje del lenguaje. En opinión de Goodman y Goodmann (1990), mantener ese equilibrio entre convención e invención debe ser uno de los factores más importantes en el desarrollo de la lectura y la escritura en las aulas. Si se logra, los estudiantes se consideran participantes de las experiencias que allí se llevan a cabo, en el caso contrario, quedarán excluidos de las experiencias literarias. Si todos ellos están sujetos a un currículum rígido y a una enseñanza intervencionista, algunos sobrevivirán y otros, por el contrario, se autoexcluirán.

8) El alumno, en las condiciones ambientales $y$ de mediación adecuadas, puede adquirir por si mismo las habilidades que necesita. La perspectiva tradicional asume que todos los alumnos deben aprender las actividades propuestas al mismo tiempo y en el mismo orden. Desde el enfoque del lenguaje integrado la planificación del maestro está influida por los intereses de los niños y, además, se busca que los alumnos puedan planificar su propio trabajo y tomar decisiones acerca de como lograr los objetivos propuestos (Froese, 1991). Con ello se persigue, entre orras cosas, que desarrollen una motivación intrínseca hacia el aprendizaje de la lecto-escritura. Este aspecto tendrá importantes consecuencias en relación con el modo en que ha de entenderse la evaluación cuya meta es, sobre todo, informar al que aprende y sólo posteriormente serän informadas otras personas. Ello supone invertir el orden que puede ser habitual, además las calificaciones dejan de tener la misma importancia que en un sistema tradicional. A través de la autoevaluación se rompe con los estrechos límites que impone la necesidad de introducir criterios ajenos al proceso, como por ejemplo el hecho de marcar para todos los alumnos la misma meta (Watson, 1989/Noviembre). 


\section{¿CUÁLES SON LAS RAÍCES DEL LENGUAJE INTEGRADO?}

Antes de pasar a exponer «sus raíces», siguiendo de cerca las que han señalado algunos de los autores que se sitúan en esta perspectiva, conviene insistir en el hecho de que este movimiento surge, sobre todo, entre los profesores que trabajan diáriamente en el aula. Es decir, al menos en cierta medida, el lenguaje integrado aparece como un movimiento innovador y alternativo frente a quienes se acercan a las situaciones de enseñanza-aprendizaje desde perspectivas tradicionales, sobre todo aquellas que, de becho, ponen el acento en los aspectos relacionados con la transmisiónrecepción del conocimiento prescindiendo de la actividad del que aprende o del significado que para éste ha de tener aquello que se aprende. En suma, estamos ante un enfoque que surge «desde abajo", pero al que es necesario encontrar apoyos y fundamentos en enfoques y teorías que permitan justificar «las prácticas» educativas que se presentan como alternativa. Nos referiremos a ellos desde una perspectiva interdisciplinar, a saber, buscando en la pedagogía, la psicología y la lingüística.

Los inspiradores de este movimiento (Goodman, 1989/Noviembre) buscan sus primera raíces en los trabajos de John Amos Comenius (1887), especialmente en sus ideas acerca del aprendizaje del niño. Podemos señalar algunas. De acuerdo con este autor, quienes aprenden son considerados como participantes activos en el proceso, que tendrá lugar en comunidades donde el que aprende decide aquello que ha de aprender y para lograrlo establecerá relaciones entre lo nuevo y lo que ya sabe. Los niños se acercan a la nueva información a través de sus experiencias vitales, manipulando objetos concretos y utilizando el lenguaje para hablar de aquello que aprenden. En este contexto, el maestro es visto como alguien que aprende junto al estudiante, todo ello en un entorno democrático en el que ambos están de acuerdo en las metas que se pretenden lograr, juntos planifican las experiencias de aprendizaje que elaboran a partir de la experiencia y de los conocimientos previos de quienes aprenden. Los maestros tratan de comprender las expectativas de los alumnos, sus culturas y las comunidades en las que viven. Los maestros, además, son conscientes de que los niños no siempre aprenden lo que ellos les enseñan, se considera que es el que aprende quien en definitiva tiene el control de lo que se enseña.

Quienes se sitúan en la perspectiva del lenguaje integrado han rastreado también en los trabajos de John Dewey $(1938 ; 1943)$. De su trabajo resaltan la necesidad de lograr un «currículum integrado», si en la vida del niño el conocimiento no se organiza en torno a un conjunto de estratos independientes las materias del currículum deben presentarse como un todo integrado. También Dewey destacó que el lenguaje es uno de los instrumentos de los que se sirve el niño para aproximarse al currículum. Así, el lenguaje oral, la lectura y la escritura han de ser enseñados sobre esa base, es decir, en cuanto que permiten al niño dar cuenta de sus experiencias y aprender de las experiencias ajenas. Teóricos posteriores en los años sesenta (por ejemplo, Taba, 1950; 1962), han insistido también en la necesidad de situar al niño ante un currículum integrado, que va allá de las relaciones que puedan establecerse entre las distinta materias e insiste en la necesidad de integrar actitudes y valores en el desarrollo del conocimiento, todo ello en el marco de una sociedad democrática.

También en la psicología han buscado sus raíces quienes trabajan en el entorno del lenguaje integrado y se fijan, sobre todo, en las perspectivas de Piaget y Vygotky. De acuerdo con un enfoque piagetiano aceptarán la idea de que el sujeto participa activamente en el desarrollo del conocimiento, los niños desarrollan sus propios procesos de conceptualización, que a menudo contradicen las versiones de los adultos. Así, los niños han de participar activamente en la comprensión del mundo, intentando resolver los problemas que éste les plantea. Fijándose en la perspectiva vygotskiana prestan especial atención a la noción de zona del desarrollo próximo, insistiendo 
en la importancia del papel del maestro, incluso aunque los que aprenden sean responsables de su propio desarrollo conceptual. Se insiste, además, en la importancia la interacción con los iguales y, especialmente, en el juego, en cuanto que facilita la creación de zonas de desarrolla próximo permitiendo al niño ir más allá de la zona de desarrollo actual.

Ya hemos señalado que las raíces del lenguaje integrado se buscan también en la lingüística y, especialmente en los trabajos de Hallyday (1975). De este autor se consideras, especialmente, sus aportaciones al papel del contexto y la situación en el aprendizaje y uso del lenguaje. En sus trabajos pueden apoyarse las discusiones acerca de de las experiencias instruccionales relacionadas con el uso natural del lenguaje en el aula. De sus aportaciones destacan el hecho de haber desarrollado un sistema de gramática funcional que relaciona el estudio del lenguaje a las acciones dentro de un contexto situacional, teniendo en cuenta incluso las relaciones entre quienes participan de la situación. También dicen inspirarse en Hallyday cuando consideran que al mismo tiempo que los niños usan el lenguaje lo aprenden y, además, aprenden sobre él (Pinnell \& Haussler, 1988). Siguiendo esta línea, el lenguaje integrado explora caminos diversos para acercarse al aprendizaje de la lectura y la escritura. Ambas han de enseñarse en situaciones reales y verdaderamente significativas para el lector o el escritor. Por lo que se refiere a la lectura, estos autores han seguido de cerca las propuestas de Rosenblatt (1938/1976) y Smith \& Goodman (1971) que la describen como un intercambio entre el lector y el texto, estableciendo los derechos de los lectores a trabajar sobre su propio significado La gente aprende a leer leyendo, vienen a decirnos. De esta forma quieren explicar el éxito de los programas en los que los estudiantes se ven inmersos en libros de lectura reales o en los que los niños que leen desde muy pequeños en su casa tienen posteriormente un mayor éxito lector. Considerando autores más recientes hay que resaltar la influencia de Don Graves (1983), que se ocupa de cómo los niños aprenden a escribir en situaciones concretas y, sobre todo, de cómo favorecer el desarrollo de la escritura en las aulas acercando ese proceso al mundo real y adoptando una perspectiva funcional.

\section{LENGUAJE INTEGRADO: UNA CORRIENTE INNOVADORA ENTRE OTRAS}

Pero para comprender un movimiento pedagógico innovador es imprescindible situarlo en el entorno en que aparece. Por ello será necesario establecer relaciones entre el lenguaje integrado y otros enfoques educativos que se han caracterizado también por sus rasgos innovadores.

\section{El lenguaje integrado en el contexto anglosajón}

En el contexto de las corrientes angloamericanas Altwerger, y colaboradores (1987/Noviembre) lo diferencia de otras experiencias que han concedido un papel relevante al lenguaje. Tal vez la más significativa sea la que se conoce como «Lenguaje basado en la experiencia, "Language Experience Approach" (por ejemplo, Peterson, 1981/Otoño). Ambos movimientos aceptan la idea de considerar la lectura como un acto personal en el que el lector se compromete con el texto, también coinciden a la enorme importancia que se concede a la literatura en el proceso de aprendizaje de la lecto-escritura. Sin embargo existen diferencias importantes entre ellos que se refieren, fundamentalmente, a las relaciones que se establecen entre el lenguaje oral y escrito. Quienes se sitúan en la perspectiva del Lenguaje basado en la experiencia consi- 
deran a la lengua escrita como un sistema derivado del lenguaje oral. Recordemos que para el lenguaje integrado el lenguaje oral y escrito están estructuralmente relacionados de forma que no puede considerarse que uno es un sistema simbólico alternativo del otro. Existen también algunos desacuerdos en otros aspectos más concretos, por ejemplo, en relación con el modo en que se utilizan algunas técnicas pedagógicas, como la reflexión y el diálogo. Mientras quienes toman la perspectiva del Lenguaje basado en la experiencia las trivializan, poniéndolas al servicio de aspectos muy puntuales como, por ejemplo, la enseñanza de la puntuación, en el lenguaje integrado esas habilidades se subordinan a una comprensión inmersa en la interacción entre el texto y el lector.

También Altwergery sus colaboradores (1987/Noviembre) lo diferencian del movimiento conocido como la Escuela abierta (Open classroom) que se apoya, entre otros autores, en las ideas de Neill (1960), pero con importantes desarrollos posteriores en algunas escuelas norteamericanas. Esta perspectiva coincide con el lenguaje integrado en el carácter activo que se asigna al que aprende, centrando el proceso de enseñanza-aprendizaje en el niño. Pero existen también entre ellos algunas diferencias. En primer lugar, la importancia que se concede al proceso de aprendizaje, frente al contenido, es quizás más importante en la escuela abierta donde, por ejemplo, se insiste menos en las materias del currículum que en la función que desempeña el contexto social del aprendizaje. También es distinto el papel que ambos movimientos conceden al maestro. Mientras que en la escuela abierta éste es un facilitador del aprendizaje, quien aporta el entorno adecuado, en el lenguaje integrado el maestro es considerado, esencialmente, como alguien que aprende con el niño en un proceso de construcción conjunta del significado.

\section{El lenguaje integrado frente a algunos movimientos innovadores de habla francesa}

En el contexto de la escuela en lengua francesa cabe destacar, entre otros, los trabajos que se han llevado a cabo en el equipo de didáctica de las lenguas de la universidad de Ginebra, siguiendo de cerca las aportaciones de Bronckart (1987) y sus colaboradores. Esta aproximación ha sido dada a conocer a los lectores de lengua castellana a través de una monografía reciente de CL\&E (Pujol, 1994a). En el artículo introductorio Pujol (1994b) establece los principios básicos de este enfoque desde una triple perspectiva. Epistemológicamente estos autores se aproximan a los principios de la psicología sociocultural, inspirada sobre todo en las ideas de Vygotsky acerca de la naturaleza social del lenguaje. Considerando algunos de sus principios básicos, por ejemplo, el hecho de que «la diversidad de discursos se explica por la infinidad de acciones verbales existentes" podríamos pensar que esta perspectiva tiene algunos aspectos en común el lenguaje integrado. Pero hay entre ellos importantes desacuerdos que se reflejan, incluso, en sus principios pedagógicos más concretos. Esas diferencias se refieren, por ejemplo, al modo en que se estructuran las secuencias didácticas. Para quienes trabajan desde el enfoque de la escuela de Ginebra,

«la enseñanza/aprendizaje de la lengua se inscribe en un proyecto pedagógico integrado, con una serie de objetivos de aprendizaje y una secuencia didáctica compuesta por diferentes módulos, cada uno de ellos con una finalidad y un objetivo específicos y bien definidos. Cada módulo contiene ejercicios relativos a cada una de las dimensiones discursivas en las que el trabajo integrado sobre los textos se alcerna con un trabajo local sobre las unidades lingüísticas» (Pujol , 1994b, p. 11).

Frente a esta tipo de enseñanza, centrada en aspectos específicos del discursos, el lenguaje integrado propone una aproximación holista, de acuerdo con la cual el len- 


\section{6}

guaje se aprende a través de su uso. En cualquier caso, CL\&E ofrece al lector español la posibilidad de contrastar ambas aproximaciones.

\section{Lenguaje integrado y movimientos innovadores en lengua castellana}

Nos referiremos, finalmente, a las posibles relaciones entre el lenguaje integrado y otros movimientos innovadores en lengua castellana. No sabemos hasta qué punto los principios que recoge el «Diseño Curricular Base», en el que se apoya la reciente reforma de la escuela, recogen un sentir general entre nuestros enseñantes. En cualquier caso, es interesante contextualizar en su marco las aportaciones del lenguaje integrado.

Hemos de reconocer que algunos de los supuestos básicos que están tras el diseño curricular, que actualmente está presente en nuestro país, no difieren en exceso de los que pueden aceptar quienes trabajan desde el enfoque del lenguaje integrado y que tienen también sus raíces en posiciones constructivistas, que priman el papel activo del que aprende.

«El lenguaje (se nos dice en el «Diseño Curricular Base») se convierte en un instrumento fundamental para construir una representación del mundo más o menos compartida y esencialmente comunicable, en un instrumento básico para la construcción del conocimiento, para la realización de aprendizajes y para el logro de una plena integración social y cultural» (M.E.C., 1989, p. 261).

Se insiste tanto en las funciones comunicativas como expresivas del lenguaje y en la importancia que tiene su aprendizaje en contextos específicos. Es más, podemos incluso ir todavía más lejos y considerar que el diseño curricular base coincide con el lenguaje integrado en la idea de que «la lengua no puede separarse del discurso oral y de los textos escritos; por ello la manipulación y reflexión en/sobre los distintos elementos de la lengua parece conveniente hacerla desde los textos (orales y escritos)» (p. 262). En cualquier caso, debemos reconocer que existen también desacuerdos importantes. No podemos olvidar que la tradición en nuestras escuelas nos sitúa ante un conjunto de saberes y conocimientos compartimentalizados; esto explica, tal vez, que estemos ante una propuesta de carácter menos integrado y también el menor papel que nuestro «Diseño Curricular Base» concede al lenguaje en el conjunto del currículum.

Existen otras aproximaciones a la enseñanza de lecto - escritura en nuestro mundo de lengua castellana en relación con las cuales será interesante contextualizar el lenguaje integrado. No pretendemos sintetizar toda ellas, sino simplemente aludir a algunas a título de ejemplo. En términos generales podemos reconocer que hay una aproximación etnográfica bien representada, por ejemplo, en los trabajos de Elsie Rockwell (1991; 1994). A nuestro modo de ver, y ello es siempre el resultado de una interpretación personal, aun coincidiendo en algunos aspectos básicos con el enfoque del lenguaje integrado, por ejemplo, en la importancia concedida al contexto en la generación del discurso, existen algunas diferencias básicas que se refieren, sobre todo, al modo en que se abordan las relaciones entre la lengua oral y escrita. Podemos decir que más que dos dimensiones de un mismo proceso, la lengua oral se considera como un instrumento básico en la interpretación de la escrita. Es esta idea de «re-interpretación" y «re-construcción» la que nos parece más interesante del trabajo de esta autora y la que, en cierto modo, va más allá del lenguaje integrado. Dicha reinterpretación, por otra parte, es inseparable del contexto social en el que se genera. Veamos, por ejemplo, cómo se plantea esa relación en el contexto del aula: 
texto y de la dinámica oral. En la relación bipolar maestro-alumnos, el rexto presente es un tercer elemento a que ambos tienen que atender; en este sentido se "triangula» la relación. por una parte la presencia del texto incide en lo oral, de hecho obliga a modificar las pautas de interacción y la lógica del contenido. Por orra parte, la dinámica oral incide en la interpretación del texto; como plantea Heath, lo «describe, repite, refuerza, amplía,enmarca o contradice" (Rockwell, 1991, p. 32)

En definitiva diríamos que desde este enfoque el lenguaje oral y escrito se complementan de forma que uno de ellos permite la re-interpretación del otro. Frente a este postura, el lenguaje integrado insiste en la continuidad más que en la complementariedad.

También en el marco de los trabajos en lengua castellana son bien conocidos en nuestro país los ya clásicos de Ferreiro y Teberosky (1979) o los más recientes de Teberosky (1992a; 1992b). Estas autoras han trabajado en profundidad no sólo el desarrollo de la capacidad de escribir sino también las ideas que tienen los niños acerca de la escritura. Se aproximan a la lecto-escritura rompiendo enfoques tradicionales, demasiado centrados en el aprendizaje mecánico y no significarivo, y consideran que la escritura es algo más que un «objeto escolar», el mundo escrito junto junto a la lengua oral forman parte de la realidad social del niño. Entre los presupuestos que parecen regir su trabajo en la actualidad hay que destacar que se defiende, ante todo, la peculiaridad de la lengua escrito. Ello se revela, por ejemplo, en su concepto de «competencia literaria». "Competencia», nos dice Teberosky, en cuanto que forma parte de los conocimientos implícitos que no pueden ser evaluados en situaciones de observación directa, y «literaria» porque no se deriva del uso conversacional del lenguaje, sino de las experiencias de interpretar, producir, comparar, evaluar el lenguage de los textos o los libros impresos. Además, y ello tiene también gran importancia, para estas autoras la didáctica del lenguaje escrito exige algo más que una aproximación sólamente funcional. En suma, estamos de nuevo ante autoras que prefieren referirse a la complementariedad entre lo oral y lo escrito más que a una continuidad entre ambos.

\section{Lenguaje integrado: breves reflexiones ante el futuro}

Pero ¿hacia dónde va el enfoque conocido como lenguaje integrado? ¿cuál es su agenda para el futuro? Goodman (1992) señala como tarea pendiente la necesidad de seguir avanzando en los principios en los que se apoya el programa: lo esencial es llevar a cabo una lectura y escritura funcionales y significativas. La literatura es un pilar en el que se apoya el aprendizaje de la lectura, la enseñanza y el aprendizaje de la escritura han de ser actividades fundamentales de la escuela; los maestros se ven reforzados en su actividad, la tiranía de los libros de texto se rompe.

Haste (1989/Noviembre) en un trabajo que él mismo titula el futuro del lenguaje integrado señala también algunos aspectos que han de tenerse en cuenta en el futuro. En primer lugar, se refiere a la necesidad de avanzar en la teoría. Los cambios suponen que la lectura y la escritura se consideran como instrumentos para el aprendizaje más que como fines en sí mismos. En este sentido, es posible orientarse hacia todo el proceso de aprendizaje a partir de la lectura y la escritura, por ejemplo, sería necesario avanzar hacia como enseñar ciencias y matemáticas a partir de ellas. Por ello, todas las áreas del currículum se ven afectadas. Se trata de ver, en definitiva, el papel que el lenguaje juega en el aprendizaje en el conjunto del currículum. En segundo lugar se refiere a la necesidad de avanzar en el conocimiento, ello significa que desde una teoría del lenguaje se ha de pasar a una teoría del aprendizaje. Su punto de partida es lo que los niños traen a la escuela. El aprendizaje comienza estableciendo conexiones con las experiencias vitales, y ello con independencia de lo que quiera 
enseñarse. Finalmente se proponen cambios en el curriculum, éste es un punto de partida para «mantener conversaciones» o comenzar entre maestros y alumnos procesos de construcción del conocimiento.

Como todo movimiento pedagógico, tras el que además se esconde una filosofía, el lenguaje integrado no está libre de críticas (Thompson, 1992). En primer lugar, podemos preguntarnos hasta que punto los estudiantes aprenderán simplemente leyendo y escribiendo y recibiendo únicamente instrucción de los maestros de forma accidental. Frente a esta idea muchos de los críticos han señalado que los niños con dificultades especiales de aprendizaje tienen más dificultades que sus compañeros para progresar cuando se ven inmersos en esta metodología. Otra importante crítica es que lenguaje integrado carece de una base profunda que, apoyada en la investigación, permita sustentar los principios en que se apoya. En este sentido se basaría, únicamente, en la práctica de los docentes. De todas estas cuestiones, y de otras muchas, nos ocuparemos en los próximos artículos que se incluyen en este número monográfico.

\section{Referencias}

Altwerger, B,; Edelsky, C., y Flores, B. M. (1987/Noviembre). Whole Language: What's new? The reading teacher, 41(2), 144-155.

Bergeron, B. S. (1990). What does the term whole language mean? Constructing a definition from the literature. Joumal of Reading Behavior, XXII (4), 301-329.

BRONCKART, J. P. (1987). Interactions, discours, significations. Langue française, 74, 29-50.

Butler, S. (1991). The writing connection. In V. Froese (Eds.), Whole-language. Practice into theory (pp. 97-148). Boston y London: Allyn and Bacon.

COMEnIUS, J. A. (1887). The orbis pictus. Siracuse, NY: C. W. Bardeen.

DEWEY, J. (1938). Experience in education. New York: Collier.

DEWEY, J. (1943). The child and the curriculum and the school and society. Chicago: University of Chicago Press.

Ferreiro, E., y Teberosky, A. (1979). Los sistemas de escritura en el desarrollo del niño. Mexico: Siglo XXI.

GOODMAN, K. S. (1992). Why whole language is today's agenda in education. Language Arts, 69, 354363.

GoOdman, K. S. (1992/Noviembre). I didn't found whole language. The Reading Teacher, 46(3), $188-$ 198.

GoOdman, Y. (1989/Noviembre). Roots of the whole language movement. The Elementary School Journal, 90(2), 113-127.

Goodman, Y. M., y GoodmanN, K. S. (1990). Vygotsky in a whole-language perspective. In L. C. Moll (Eds.), Vygotsky and education (pp. 223-250). Cambridge: Cambridge University Press.

Graves, D. (1983). Writing Teachers and children at work (Trad. cast Didáctica de la escritura. Madrid: Morata-MEC, 1991). Portsmouth, NH: Heinemann.

Halliday, M., y HaSAN, R. (1976). Cohesion in English. London: Longman.

Hallyday, M. A. (1975). Leaming bow to mean. New York: Elsevier North-Holland.

HASTE, J. C. (1989/nNoviembre). The future of whole language. The Elementary School Journal, 90(2), 242-249.

M.E.C. (1989). Diseño curricular base. Educación primaria. Madrid: M.E.C.

NeIL, A. S. (1960). Summerbil: A radical approach to child rearing. New York: Hart Publishing.

Peterson, R. L. (1981/Otoño). Language experience: A methodic approach to teaching literacy. Georgia Journal of Reading, 7, 15-23.

PINNELL, G., y HAUSSLER, M. (1988). Impact of language research on curriculum. Newark, DE: International Reading Association.

Pujol, M. (1994a). La didáctica de la lengua oral. Número Monográfico. Comunicación, Lenguaje y Educación, 23, 5-116.

Pujol, M. (1994b). La pedagogía del texto: Enseñanza/aprendizaje del producto final y del proceso de producción verbal. Comunicación, Lenguaje y Educación, 23, 9-16.

ROCKWELL, E. (1991). Palabra escrita, interpretación oral: Los libros de texto en la clase. Infancia y aprendizaje, 55, 29- 44.

ROSENBLATT, L. (1938/1976). Literature through exploration (3erd. edition). New York: Noble and Noble.

SMITH, F., y GoODMAN, K. (1971). On the psycholinguistic method of teaching reading. Elementary School Journal, 71, 177-181. 
TABA, H. (1950). Elementary curriculum in intergroup relations. Washington, DC: American Council on Education.

TABA, H. (1962). Curriculum development. New York: Harcourt, Brace \& World.

TEBerosky, A. (1992). Reescribiendo textos: producción de adulta poco escolarizadas. Infancia y aprendizaje, 58, 107-124.

ThOMPSON, R. A. (1992). A critical perspective on whole lenguage. Reaing Psychology: An International Quarterly, 13(131-155).

WATSON, D. J. (1989/Noviembre. Defining and describing whole language. The Elementary School Journal, $90(2), 129-141$.

ALTwERGER, B.; EDELSKY, C., y Flores, B. M. (1987/Noviembre). Whole Language: What's new? The Reading Teacher, 41(2), 144-155.

ROCKWELL, E. (1991). Palabra escrita, interpretación oral: Los libros de texto en la clase. Infancia y aprendizaje, 55, 29- 44.

ROCKWELL, E. (1994). Documenting orality and literacy in rural Mexico. In J. Werstch \& J. D. Ramírez (Eds.), Explorations in Socio-Cultural studies. Vol. II. Literacy and other forms of mediated action. Madrid: Fundación Infancia y Aprendizaje.

TEBEROSKY, A. (1992a). A prendiendo a escribir. Barcelona: ICE (universidad de Barcelona)/Horsori.

TEBEROSKY, A. (1992b). Reescribiendo textos: producción de adulta poco escolarizadas. Infancia y aprendizaje, $58,107-124$.

Lenguaje integrado: ¿simple práctica, un método o una filosofía?

Pilar Lacasa, José J. Anula y Beatriz Martín del Campo CL\&E, 1995, 25, pp. 9-19

Resumen: Este artículo revisa los presupuestos del «lenguaje integrado" y analiza en qué sentido puede ser considerado algo más que una simple suma de prácticas educativas; puede entenderse también como una filosofía. Se buscan además las bases que forman sus raíces. Finalmente, esta perspectiva se contextualiza en el conjunto de movimientos innovadores que han aparecido en educación en los entornos anglo-americanos, franceses y, como no, en los países de habla hispana.

Datos sobre el autor: Pilar Lacasa es Profesora Titular de Psicología Evolutiva y de la Educación. Actualmente trabaja en temas relacionados con el desarrollo, aprendizaje y enseñanza de la lecto-escritura desde un enfoque socio-cultural.

José Jerónimo Anula es Licenciado en Psicología por la Universidad de La Laguna. Actualmente es becario del Plan de Formación de Personal Docente e Investigador. Trabaja en el campo de las creencias y las actitudes y trata de acercarse a ellas a partir del análisis del discurso.

Beatriz Matín del Campo es Licenciada en Psicología por la Universidad Autónoma de Madrid. Actualmente es becaria del Plan de Formación de Personal Docente e Investigador de la U.N.E.D. Trabaja en temas relacionados con el desarrollo de la lengua escrita, le interesa especialmente el tema de los formatos textuales.

Dirección: Pilar Lacasa. Departamento de Educación. Facultad de Ciencias de la Educación. Universidad de Córdoba. Priego de Córdoba, n 4 . 14013 Córdoba. Fax: 957218923.

c) PERMISOS PARA CITAR O REPRODUCIR EN OTRAS FUENTES: Se pueden citar libremente hasta 500 palabras. Para reproducir una porción de texto mayor, figuras o ilustraciones, se deberá pedir permiso por escrito a la revista, especificando el uso al que se destina el texto. En todos los casos, se deberá citar el copyright de $C L \& E$. En el caso de artículos o textos que hayan sido a su vez reproducidos en $C L \& E$ los interesados deberán dirigirse tanto a los detentadores del copyright original como a $C L \& E$, en el caso de que se quiera hacer uso de la traducción. FOTOCOPIAS: Para todo lo relacionado con el uso mediante fotocopia del material de esta revista, deberán dirigirse a: CEDRO, C/ José Marañón, 10, 3. Izda. Tel. 5941575 . Fax 4453567 